\title{
THE X-RAY EMISSION OF QUASARS AS OBSERVED BY GINGA
}

\author{
A.J. LAWSON and M.J.L. TURNER \\ University of Leicester \\ University Rd. \\ Leicester LE1 TRH \\ United Kingdom
}

\begin{abstract}
.
We present preliminary results on a spectal analysis of quasars observed by the $\mathrm{X}$-ray observatory Ginga. Simple power-law models with fixed Galactic absorbtion provide an adequate description of the spectra for most of the sources in the 2-18 keV band. A small number of sources show evidence for a feature at $6.4 \mathrm{keV}$ (in the source rest frame) due to Fe line emission. Maximum likelihood and Spearman rank tests were used to investigate the relationship between radio loudness and X-ray spectral index in this class of object. These tests showed, respectively, that the mean X-ray spectral index of radio quiet quasars (RQQs) is significantly different from that of flat spectrum radio loud quasars (FRSQs) at the $>99 \%$ level, and that the dominant relationship with spectral index is radio loudness (not X-ray luminosity or redshift) at $>99 \%$ significance. This last result has not previously been demonstrated in this band, but agrees with findings in the lower energy Einstein band $(0.5-3.5 \mathrm{keV})^{[1]}$. These results are discussed in the context of current unified models.
\end{abstract}

Key words: galaxies:active - galaxies:nuclei - quasars:general - radio continuum:galaxies - Xrays:galaxies

\section{Method and Results}

Ginga observed over 40 quasars $\left(\mathrm{M}_{\mathrm{B}}<-23\right)$ during its $4+$ years in orbit. The data from each object were background subtracted using standard techniques ${ }^{[2]}$ and then fitted with a power-law model including hydrogen column absorbtion. There was no requirement in any of the spectra for a column greater than the galactic. Amongst observations with sufficient sensitivity, those of radio quiet objects show evidence for an iron line at $6.4 \mathrm{keV}$ (source frame) with $\sim 200 \mathrm{eV}$ equivilent width, but the radio loud objects show no such emission, with EQW $<20 \mathrm{eV}$. Reflection ${ }^{[3]}$ can be fitted to the radio quiet spectra, but is not required statistically. Spectra from 27 objects were used to investigate the relationship between X-ray spectral index and radio loudness. (N.B. Not all the objects observed are used, as some fall below the survey flux limit and others presently have unresolved problems in the background subtraction.)

Previous papers ${ }^{[1,4,5]}$ have shown a relationship between X-ray spectral slope and radio loudness in quasars, in the sense that radio loud objects have flatter spectra. There is also some evidence ${ }^{[6,7]}$ that the slope varies within the radio loud group, with the slope of steep spectrum radio quasars, SRSQs (i.e. those that are lobe dominated) being slightly greater than that of the FRSQs (core dominated objects). Figure 1 shows the plot of index versus radio loudness for this sample (Key: Cross in circle - RQQs $\left(R_{L}<1\right)$, Square - SRSQs, Star - FRSQs).

T. J.-L. Courvoisier and A. Blecha: Multi-Wavelength Continuum Emission of AGN, 123-125.

(C) 1994 IAU. Printed in the Netherlands. 


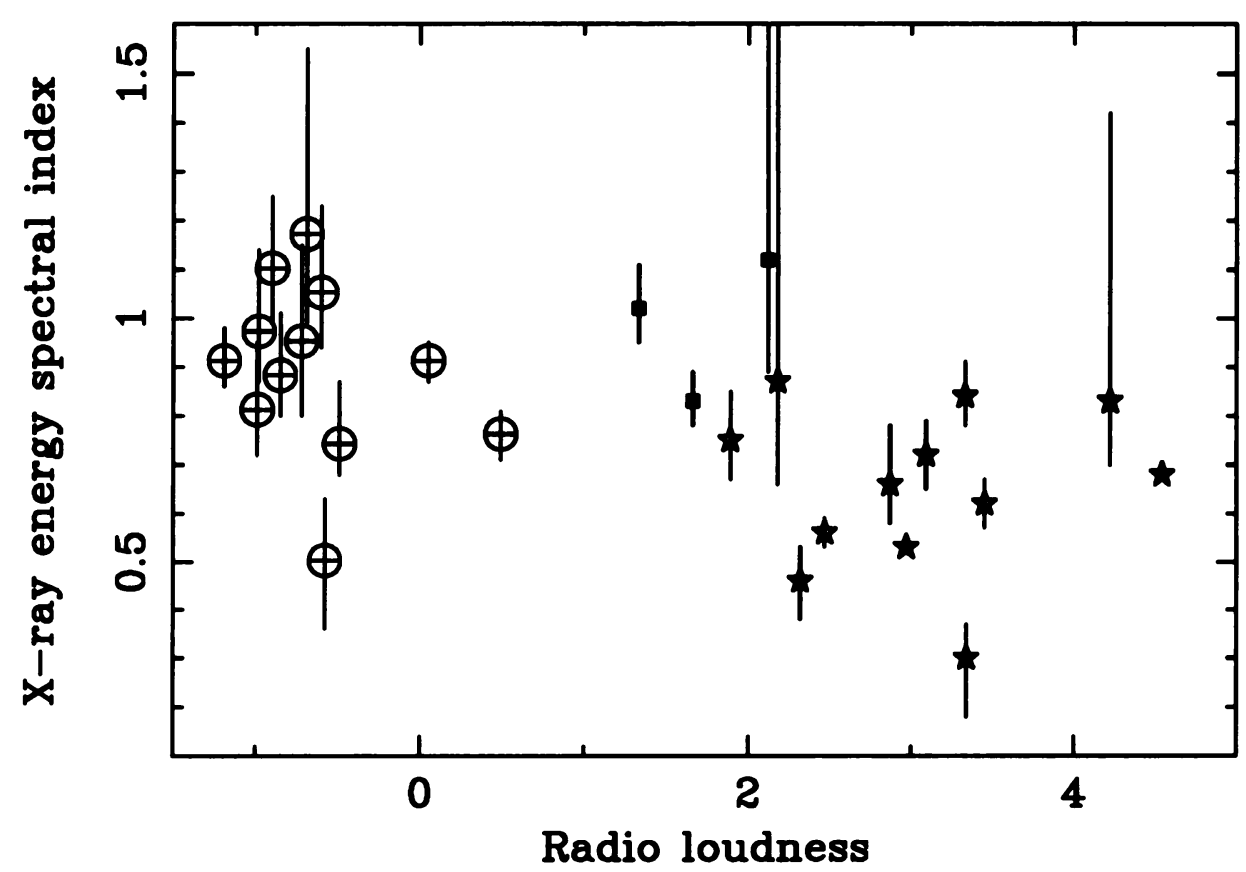

Fig. 1. Plot of X-ray spectral index vs radio loudness for the current Ginga sample

Maximum likelihood methods return the following for the mean index $(\bar{\alpha})$ and error on the three groups of interest in this study. Respectivly the results are for the RQQ, FRSQ and SRSQ groups; $0.86 \pm 0.03,0.63 \pm 0.07$ and $0.95 \pm 0.18$. These results agree well with those from the previous studies with the $\bar{\alpha}$ of the FRSQs being significantly different from that of RQQs at the $>99 \%$ level. Also the SRSQ group resembles more the RQQ group than the FRSQ group (the indices of the two radio loud groups are different at $90 \%$ significance). Using Partial Spearman tests it is shown, for the first time in the $2-18 \mathrm{keV}$ band, that the dominant relationship with $\alpha$ is that of radio loudness rather than with $\mathrm{L}_{\mathrm{X}}$ or $z$ at $>99 \%$ significance. This agrees with findings ${ }^{[1]}$ in the soft X-ray band.

\section{Discussion}

\subsection{RADIO LOUD OBJECTS}

High polarizations, superluminal motion and the EGRET detections of exclusively radio loud objects provides good evidence that relativistic jet motion and beaming are important in these objects and probably dominate the emission at high energies. Recent work ${ }^{[8]}$ has suggested that radio loud objects (FRSQs and SRSQs) 
and FRII radio galaxies could be the same objects viewed at different angles with respect to the jet, and it has also been shown that jet models are consistent with this orientation effect ${ }^{[9]}$. Modelling of the jet emission components ${ }^{[10]}$ predicts a slope of $\alpha \sim 0.5$ for the Compton component which would be dominant in the hard $\mathrm{X}$-ray band. Results of our study are consistent with the above findings; an index of 0.6 for the FRSQs, the SRSQs with a different slope to the FRSQs and the lack of a thermal iron emission line possibly due to swamping of the continuum by the doppler boosted component.

\subsection{RADIO QUIET OBJECTS}

The RQQs, with no instrinsic absorbtion column and $6.4 \mathrm{keV} \mathrm{Fe} \mathrm{line} \mathrm{emission,}$ show similarities in this respect to Seyfert galaxies. However, the spectral slopes of the RQQs are generally steeper from those of the Seyfert 1 's ${ }^{[11]}$ at $\sim 99 \%$ significance. This difference in spectral index may well be related to luminosity. ROSAT studies of $\mathrm{AGN}^{[12]}$ have shown absorbtion at a few $\mathrm{keV}$ which is thought to be due to ionised material located in the funnel created by the molecular torus. Simulating spectra at the flux levels of a typical RQQ with models with and without a warm absorber show an increase from a slope of $\sim 0.7$ to $\sim 0.9$ when the absorber is removed. This neatly corresponds to what is seen in our results, though we do not claim that this is the solution. If luminosity and size scale with mass, the only way to boost the ionization of the material is for the black hole to be accreting nearer the Eddington limit. So RQQs could be Seyfert type objects with a higher effieciency of accretion.

\subsection{CONCLUding REMARKS}

Our results add support to findings of previous studies and agree well with the current beaming and orientation unified models.

\section{References}

1. Wilkes B.J., Elvis M., 1987, ApJ, 323, 243

2. Hayashida K. et al., 1989, PASJ, 41, 373

3. Pounds K.A., Nandra K., Stewart G.C., George I.M., Fabian A.C., 1990, Nat, 344, 132

4. Lawson A.J., Turner M.J.L., Williams O.R., Stewart G.C., Saxton R.D., 1992, MNRAS, 259,743

5. Williams O.R. et al., 1992, ApJ, 389, 187

6. Shastri P., Wilkes B.J., Elvis M., McDowell J., 1993, ApJ, 410, 29

7. Worral D.M., Giommi P., Tananbaum H., Zamorani G., 1987, ApJ, 313, 596

8. Padovani P., Urry C.M., 1992, ApJ, 387, 449

9. Ghisellini G., Padovani P., Celotti A., Maraschi L., 1993, ApJ, 407, 65

10. Ghisellini G., Maraschi L., 1989, ApJ, 340, 181

11. Nandra K., 1991, PhD Thesis, University of Leicester

12. Nandra K., Pounds K.A., 1992, Nat, 359, 215 